\title{
Timing of surgery for sciatica: subgroup analysis alongside a randomized trial
}

\author{
Wilco C. Peul • Mark P. Arts · Ronald Brand • \\ Bart W. Koes
}

Received: 18 October 2008/ Accepted: 19 December 2008/Published online: 9 January 2009

(C) The Author(s) 2009. This article is published with open access at Springerlink.com

\begin{abstract}
Surgery speeds up recovery for sciatica. Prolonged conservative care with surgery for those patients with persistent sciatica however, yields similar results at 1 year. To investigate whether baseline variables modify the difference in recovery rates between these treatment strategies, baseline data of 283 patients enrolled in a randomized trial, comparing early surgery with prolonged conservative care, were used to analyse effect modification of the allotted treatment strategy. For predictors shown to modify the effect of the treatment strategy, repeated measurement analyses with the Roland Disability Questionnaire and visual analogue scale pain as continuous outcomes were performed for every level of that predictor. Presumed predictive variables did not have any interaction with treatment, while "sciatica provoked by sitting" showed to be a significant effect modifier $(P=0.07)$. In a
\end{abstract}

The authors belong to The Hague-Leiden Spine Intervention Prognostic Study (SIPS) Group.

W. C. Peul $(\varangle) \cdot$ M. P. Arts

Department of Neurosurgery, Leiden University Medical Center,

PO Box 9600, 2300 RC Leiden, The Netherlands

e-mail: w.c.peul@lumc.nl

W. C. Peul - M. P. Arts

Department of Neurosurgery, Medical Center Haaglanden,

The Hague, The Netherlands

R. Brand

Department of Medical Statistics,

Leiden University Medical Center,

Leiden, The Netherlands

B. W. Koes

Department of General Practice,

Erasmus Medical Center Rotterdam,

Rotterdam, The Netherlands
Cox model we estimated a hazard ratio (HR, surgery versus conservative) of 2.2 (95\% CI 1.7-3.0) in favour of surgery when sciatica was provoked by sitting, while the HR was 1.3 (95\% CI 0.8-2.2) when this sign was absent. The interaction effect is marginally significant (interactions are usually tested at the $10 \%$ level) but the patterns generated by the repeated measurement analyses of all primary outcomes are completely consistent with the inferred pattern from the survival analysis. Classical signs did not show any contribution as decision support tools in deciding when to operate for sciatica, whereas treatment effects of early surgery are emphasized when sciatica is provoked by sitting and negligible when this symptom is absent.

Keywords Sciatica $\cdot$ Microdiscectomy ·

Conservative treatment . Randomized controlled trial . Pain

\section{Introduction}

Sciatica is characterized by radiating pain in an area of the leg typically served by one lumbar or sacral spinal nerve root and is sometimes associated with sensory and motor deficit. Apart from infrequent causes, sciatica is mostly due to a herniated lumbar disc. Because of the high prevalence in general practice and the major impact of low back disorders on society [19], surgery is frequently performed to speed up recovery of sciatica. Probably as a result of sociocultural circumstances, different timing of surgical removal of the herniated portion of the disc appears to vary greatly in the western countries [5]. Recently the option of surgery was offered to patients after only 6 weeks of unremitting sciatica. The major reason to offer early surgery was the unattractive alternative: the slow natural course of sciatica, 
occasionally extending over 4 years $[1,24]$. Globally two options were available: (1) early surgery and (2) prolonged conservative care, possibly with surgery at a later date. Since surgery is economically affordable and relatively safe, most patients in western countries prefer early surgery rather than to wait for months or even years, risking long term work-disability and presence of chronic pain. Recently this study provided evidence that the prolonged conservative care strategy resulted in complete recovery at 1 year as similar as the surgical strategy, but it took twice as long compared to early surgery [15]. The 1-year effects of the two assigned treatment strategies were similar as far as function and pain were concerned in the randomized cohorts. The contribution of this study is that patients, opting for early surgery, now are able to base their decision on realistic data about the alternative strategy, with similar outcomes at 1 and 2 years [17]compared to early surgery. Individual decisions regarding early surgery or not are still difficult to make. Since treatment effects can differ between subgroups of patients, this might influence the indication for early disc surgery.

Therefore, it would be of great interest to patients and physicians to define determinants which occur early in the course of sciatica and predict the speed of recovery with either prolonged conservative care or early surgery. Insight in these determinants may help in the decision about when to perform surgery. We carried out a subgroup analysis of data from the aforementioned randomized trial to evaluate anamnestic, neurological and radiological variables which might in theory influence the difference in rate of recovery between the two treatment strategies.

\section{Methods}

A multicenter prospective randomized trial was designed to determine for patients with a short duration of severe sciatica, whether early surgery resulted in a more effective outcome during the first year, compared to a strategy of prolonged conservative treatment possibly with delayed surgery if indicated. The medical ethics committee at each of the nine participating hospitals approved the protocol. Written informed consent was obtained from all patients. Details of the design, study protocol and prognostic variables are previously published together with the primary outcomes [14].

\section{Patients}

Eligible patients were 18-65 years of age, with radiological confirmation of a clinically expected disc herniation causing an incapacitating lumbosacral radicular syndrome lasting between 6 and 12 weeks as documented by the attending neurologist. At the time of enrolment and randomization an independent research nurse verified persistence of complaints and surgical indication. Patients were excluded if they presented with a cauda equina syndrome or severe paresis (MRC $<3$ ). Identical complaints in the past 12 months, a history of spinal surgery, bony stenosis, spondylolisthesis, pregnancy or severe comorbidity also led to exclusion. A computer generated permutedblock scheme was used for randomization, stratified according to center ( $n=9$; see Appendix). The patients were randomized by opening an opaque envelope containing the patient's assigned strategy. Obviously it was not possible to blind patients or their physicians.

\section{Treatment}

Early surgery was scheduled within 2 weeks of assignment and only cancelled if spontaneous recovery occurred before the date of surgery. Under either general or spinal anaesthesia the herniated part of the disc was removed together with as much as possible degenerated nuclear material. Bony removal to gain access to the disc space was minimized and likewise subtotal disc excision was never pursued. The duration of the hospitalization depended on the patient's functional abilities. Since the protocol of the participating surgical departments was not changed, usual care was provided. At home the rehabilitation process was supervised by the physiotherapist on the base of a standardized exercise protocol. Patients were advised to resume their regular jobs from 6 weeks on, depending on the nature of the work. Postoperative care included out-patient control at 8 weeks or earlier if the patient worried about the course and suffered aggravation of symptoms.

Prolonged conservative management was provided by the general practitioner. Ample information was provided about the favourable prognosis. If necessary the prescription of pain medication was adjusted according to existing clinical guidelines. If there was considerable fear of movement, the help of a physiotherapist was recommended. Further on treatment was aimed mainly at resumption of daily activities. However if sciatica was still present at 6 months after randomization, microdiscectomy was offered after a repeat magnetic resonance imaging (MRI) showed the disc herniation again. Increasing drugresistant leg pain or progressive neurological deficit were reasons for performing surgery even before 6 months.

\section{Outcomes}

Functional outcome assessed by means of the Roland Disability Questionnaire (RDQ) for sciatica, intensity of leg or back pain by a $100-\mathrm{mm}$ visual analogue scale (VAS) for leg pain (VAS-leg and VAS-back) and a questionnaire 
of patient's global impression of change questionnaires on a 7-points Likert self-rating scale of recovery were filled out at 2, 4, 8, 12, 26, 38 and 52 weeks [6, 13].

For the current subgroup analyses, the patient's perceived recovery was used as dependent variable in dichotomized form, hence easily interpretable in general practice. "Very much improved" and "much improved" were coded as recovered, while "minimally improved", "no change", "minimally worse", "much worse" and "very much worse" were coded as not recovered. This global impression of change was recorded during every follow-up moment during the first year. The outcome variable under study is the time till first occurrence of "recovery" as defined above.

Prognostic variables

Possibly prognostic determinants were selected on the basis of classical physiological hypotheses or resulted from earlier studies. These socio-demographic, symptom, neurological and radiological variables (Table 1) were collected before randomization was performed.

\section{Statistical analysis}

Data collection and quality checks were performed using the ProMISe data management system of the Department

Table 1 Predefined prognostic variables

Demographic variables
Age $<40$ years versus $\geq 40$ years
Intellectual versus physically demanding job
Anamnestic and neurological variables
Acute start LSRS versus slow start
History of back pain versus no history
Influence of coughing, sneezing on complaints versus no influence
Difficulty to put on shoes and/or socks versus no difficulty
Straight leg raising $\leq 60^{\circ}$ versus $>60^{\circ}$
Positive crossed straight leg raising sign versus negative sign
VAS-pain $>70$ mm versus $<69$ mm
Tingling/numbness in pain area versus no tingling
Pain leg worse by sitting versus no worsening
McGill affective high score versus low score
Radiological variables
MRI disc sequester versus contained disc herniation
MRI circumferential gadolinium enhancement versus no
enhancement of disc herniation
Mediolateral versus median and lateral disc herniation
Miscellaneous variables
Preference for surgery versus no preference for surgery
Disc herniation at L5S 1 versus L4L5

of Medical Statistics and Bioinformatics of the LUMC. All statistical analyses were carried out using SPSS version 14.0 .

The outcome "recovery" and the time until this event occurred, were studied in the framework of survival analysis. A Cox proportional hazards model was used in all analyses. Effect modification of each predictor was tested in a model containing the treatment allocation, the predictor and the interaction between them. If the $P$-value of the interaction term was $<0.10$, the interaction was classified as "significant" in view of the lower power of such interaction tests. If a predictor was shown in this way to exert an effect modification on the binary outcome "recovery" the same model (two main terms + interaction) was specified in a repeated measurements analysis of variance for two continuous outcomes (RDQ and VAS). Here the approach was not to formally test the significant, but to estimate the outcome in the strata created by the interaction to verify whether a consistent behaviour was presented between dichotomous and continuous outcomes. Following the analysis of the interaction effects, explorative Cox regression analyses of other basic demographic, neurological and radiological variables, chosen because of a clinically plausible relationship to outcome measures, were carried out.

\section{Results}

Baseline demographic and neurological variables did not differ between groups. The unadjusted hazard ratio (HR) as estimated in a univariable Cox model with recovery as endpoint was 2.0 (95\% CI 1.7-2.2), favouring early surgery (Fig. 1a). Bivariate testing of the predefined prognostic variables showed a significant interaction effect of "sciatica provoked by sitting" with the "treatment strategy" $(P=0.07)$, but no significant interaction effect of any of the other predefined variables was found (Table 2). Interestingly the presumed influence of classical neurological tests on speed of recovery could not be confirmed and, in contrast to former medical beliefs interactions were even absent, showing equal recovery rates for different levels of these variables. Treatment preference of patients did not show any interaction with early surgery either.

A survival model with "treatment-by-randomization", "sciatica provoked by sitting" as well as their interaction, showed a differential effect on rate of recovery (Fig. 1b, c). A survival model without the treatment variable as an independent variable and thus only containing the presence or absence of sciatica provoked by sitting, did not provide any prognostic value for the speed of recovery rates. Patients with sciatica provoked by sitting did experience a slower rate of recovery when randomized to prolonged 
Panel A Unadjusted Survival Curves [17]

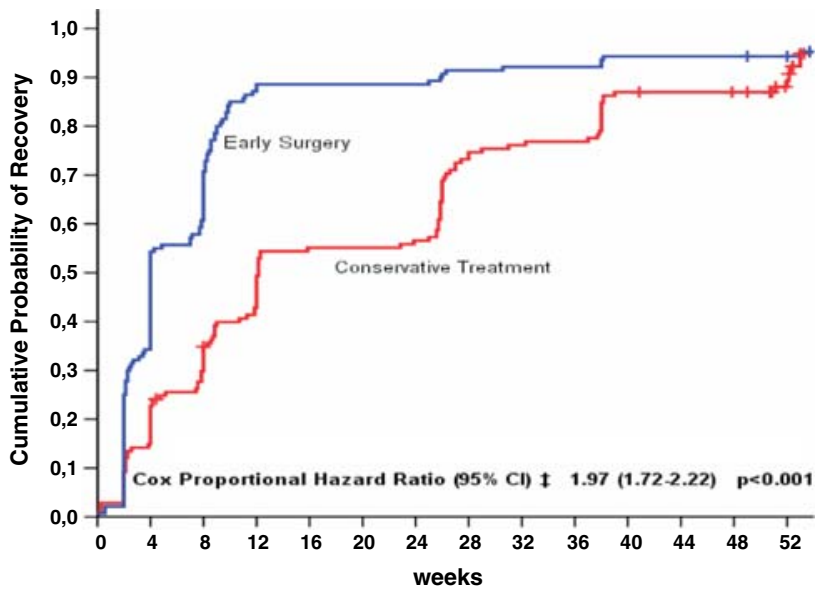

Panel B Sciatica not provoked by sitting

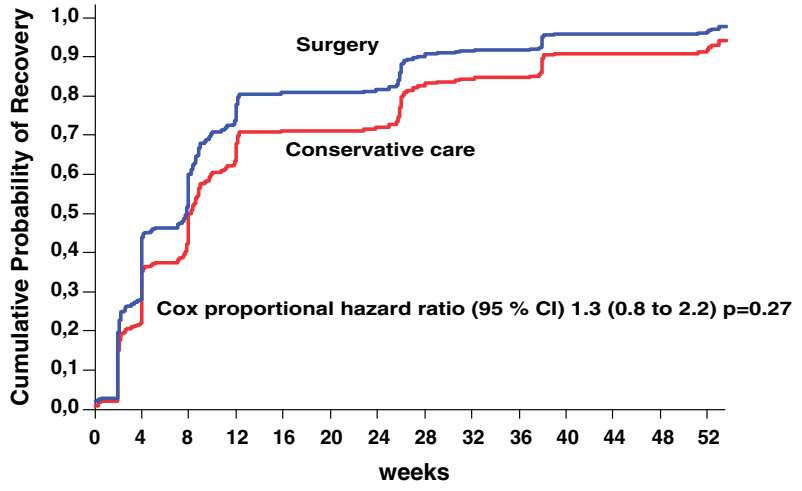

Panel C Sciatica provoked by sitting

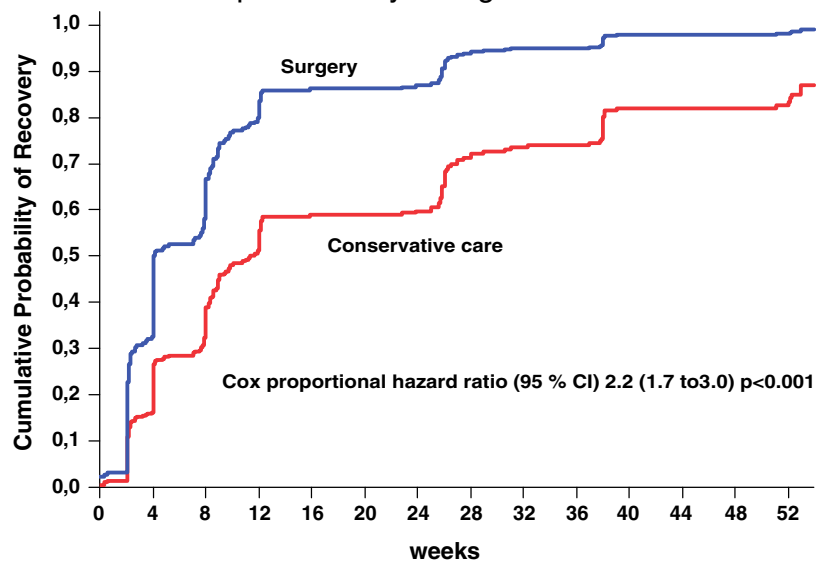

Fig. 1 Cox proportional hazard analyses. Panel a presenting the original unadjusted curves [17], while panels $\mathbf{b}$ and $\mathbf{c}$ represent stratified analyses, for sciatica not provoked by sitting and sciatica provoked by sitting, respectively

conservative treatment while surgery accelerated the rate of recovery with an estimated HR of 2.2 (95\% CI 1.7-3.0). When patients did not experience leg pain provoked by sitting, the survival curves for both randomized treatment strategies come close together, corresponding with similar average speed of recovery rates: HR 1.3 (95\% CI 0.8-2.2). Repeated measurement analysis, stratified by "sciatica provoked by sitting", gave similar findings with RDQ and VAS pain outcomes showing diverging curves when sitting provoked sciatica. Areas under the RDQ and VAS back pain curves over the first year of early surgery compared to conservative treatment were even statistically different ( $P=0.05$ and 0.03 , respectively) in contrast to the original analysis without stratifying variables. These outcomes over the first year between early surgery and conservative treatment did not show relevant differences when sciatica was not provoked by sitting and early surgery even gave less favourable results during the first months compared to conservative treatment in this group (Fig. 2).

\section{Discussion}

This randomized trial unequivocally showed that early surgery led to significantly faster recovery compared to prolonged conservative care but failed to present any interaction with (effect modification by) classical neurological signs and MRI findings. Remarkably only the anamnestic finding "sciatica provoked by sitting" showed interaction with timing of surgery, and thus influenced rate of recovery. These results were markedly consistent in stratified analyses of all primary outcomes over the first year and presented a larger effect size in favour of early surgery in those patients who were unable to sit as a result of sciatic neuralgia.

The finding that classic physical signs and high preference of the patient for surgery did not affect the results of treatment strategies was surprising and not expected. Currently most physicians and physiotherapists refer patients for surgery under the near mandatory condition that the straight leg raise test provokes sciatica [18]. The design of this trial made it possible to include enough patients with a negative straight leg raising test. These, however, form a minority which may be due to selection bias. Therefore these results must have to be interpreted very carefully, which also holds true for the findings regarding patient preferences. Earlier prognostic studies suggested a realistic relationship between patient's and doctor's preferences and expectations on the one hand and outcome on the other [11]. These expectations are still likely to play a major role since the patients in this randomized trial were all eager to undergo surgery; in fact this was their main motive to visit the outpatient clinic of the participating hospitals. Only a minority of patients did not have a clear preference for surgery and no patient had a preference for the conservative treatment strategy. Therefore the lack of influence of patient preferences on treatment strategies is not directly applicable to general practice. 
Table 2 Mean hazard ratios, with their lower to upper 95\% CI for all predefined variables and their interaction with early surgery compared to prolonged conservative treatment with possible delayed surgery

\begin{tabular}{|c|c|c|c|c|c|}
\hline Subgroup & Proportion \% & Lower & Mean & Upper & $\begin{array}{l}P \text {-value } \\
\text { interaction }\end{array}$ \\
\hline Overall & 100 & 1.72 & 1.97 & 2.22 & \\
\hline \multicolumn{6}{|l|}{ Age } \\
\hline$<40$ Years & 41 & 1.69 & 2.50 & 3.66 & \multirow[t]{2}{*}{0.12} \\
\hline$\geq 40$ Years & 49 & 1.21 & 1.68 & 2.32 & \\
\hline \multicolumn{6}{|l|}{ Intellectual job } \\
\hline Non-intellectual & 36 & 1.21 & 1.88 & 2.92 & \multirow[t]{2}{*}{0.83} \\
\hline Intellectual & 64 & 1.45 & 2.00 & 2.76 & \\
\hline \multicolumn{6}{|l|}{ Physical demanding work } \\
\hline Non-physical & 61 & 1.29 & 1.80 & 2.51 & \multirow[t]{2}{*}{0.61} \\
\hline Physical demanding & 39 & 1.37 & 2.06 & 3.1 & \\
\hline \multicolumn{6}{|l|}{ Sex } \\
\hline Male & 66 & 1.57 & 2.12 & 2.87 & \multirow[t]{2}{*}{0.64} \\
\hline Female & 34 & 1.20 & 1.87 & 2.92 & \\
\hline \multicolumn{6}{|l|}{ Start sciatica } \\
\hline Acute severe & 61 & 1.40 & 1.94 & 2.68 & \multirow[t]{2}{*}{0.91} \\
\hline Slowly increasing & 39 & 1.27 & 1.89 & 2.79 & \\
\hline \multicolumn{6}{|l|}{ Influence intra-abdominal pressure } \\
\hline Provocation sciatica & 73 & 1.57 & 2.10 & 2.81 & \multirow[t]{2}{*}{0.45} \\
\hline No provocation & 27 & 1.06 & 1.70 & 2.74 & \\
\hline \multicolumn{6}{|l|}{ Lasègue's sign } \\
\hline Straight leg raising $>60^{\circ}$ & 25 & 1.17 & 1.92 & 3.15 & \multirow[t]{2}{*}{0.88} \\
\hline Straight leg raising $\leq 60^{\circ}$ & 75 & 1.50 & 2.01 & 2.70 & \\
\hline \multicolumn{6}{|l|}{ Crossed straight leg raising } \\
\hline Negative & 41 & 1.11 & 1.61 & 2.34 & \multirow[t]{2}{*}{0.17} \\
\hline Positive & 59 & 1.64 & 2.28 & 3.18 & \\
\hline \multicolumn{6}{|l|}{ VAS leg pain intensity } \\
\hline$>70$ & 54 & 1.35 & 1.94 & 2.79 & \multirow[t]{2}{*}{0.98} \\
\hline$\leq 70$ & 46 & 1.37 & 1.93 & 2.71 & \\
\hline \multicolumn{6}{|l|}{ Sciatica provocation by sitting } \\
\hline No provocation & 24 & 0.80 & 1.30 & 2.2 & \multirow[t]{2}{*}{0.07} \\
\hline Provocation & 76 & 1.70 & 2.24 & 2.99 & \\
\hline \multicolumn{6}{|l|}{ McGill affective scores } \\
\hline Low score $<3$ & 49 & 1.34 & 2.05 & 3.00 & 0.60 \\
\hline High score & 51 & 1.47 & 1.90 & 2.46 & \\
\hline MRI sequester & & & & & \\
\hline Contained disc herniation & 59 & 1.40 & 1.96 & 2.74 & 0.81 \\
\hline Sequester & 41 & 1.23 & 1.84 & 2.75 & \\
\hline MRI gadolinium & & & & & \\
\hline No enhancement & 34 & 1.425 & 2.32 & 3.77 & 0.60 \\
\hline Enhancement & 66 & 1.38 & 1.97 & 2.83 & \\
\hline MRI level disc herniation & & & & & \\
\hline L5S1 & 61 & 1.39 & 1.93 & 2.67 & 0.75 \\
\hline L4L5 or L3L4 & 39 & 1.19 & 1.77 & 2.64 & \\
\hline Preference for surgery & & & & & \\
\hline Strong preference for surgery & 39 & 1.39 & 2.07 & 3.09 & 0.73 \\
\hline Some or no preference & 61 & 1.38 & 1.90 & 2.61 & \\
\hline Tingling/numbness pain area & & & & & \\
\hline No sensation & 10 & 1.1 & 2.3 & 5.1 & 0.66 \\
\hline Sensation & 90 & 1.5 & 1.9 & 2.5 & \\
\hline
\end{tabular}


Fig. 2 Repeated measurement analysis curves of mean scores for Roland Disability

Questionnaire (panel a), leg pain (panel b) and back pain (panel c) on a visual analogue scale stratified for "sciatica provoked by sitting (the mean difference between areas under the curves are expressed by the corresponding $95 \% \mathrm{CI}$ ). All three panels show the 52-week curves with $95 \%$ CI represented by vertical bars at consecutive moments of measurement. Red lines represent the conservative treatment group, while the blue lines represent early surgery.

Areas under the curve $(A U C)$ are described by their mean \pm SE. Panel a represents the mean disability scores at consecutive moments of measurement stratified for sciatica provoked by sitting. The overall difference between the areas under the curves over 12 months is not significant for sciatica not provoked by sitting $(P=0.77)$ and significant for provoked by sitting $(P=0.05)$ in favour of early surgery. Panel b represents mean visual analogue scores for intensity of leg pain in millimetre. The difference between the mean AUC's is not significant for sciatica not provoked by sitting $(P=0.70)$ and significant for sciatica provoked by sitting $(P<0.001)$ in favour of early surgery. Panel $\mathbf{c}$ represents mean visual analogue scores for intensity low back pain in $\mathrm{mm}$. Starting with a lower intensity score when compared to leg pain, the mean AUC's exhibit no significant difference for sciatica not provoked by sitting $(P=0.47)$ and significant for sciatica provoked by sitting $(P=0.03)$
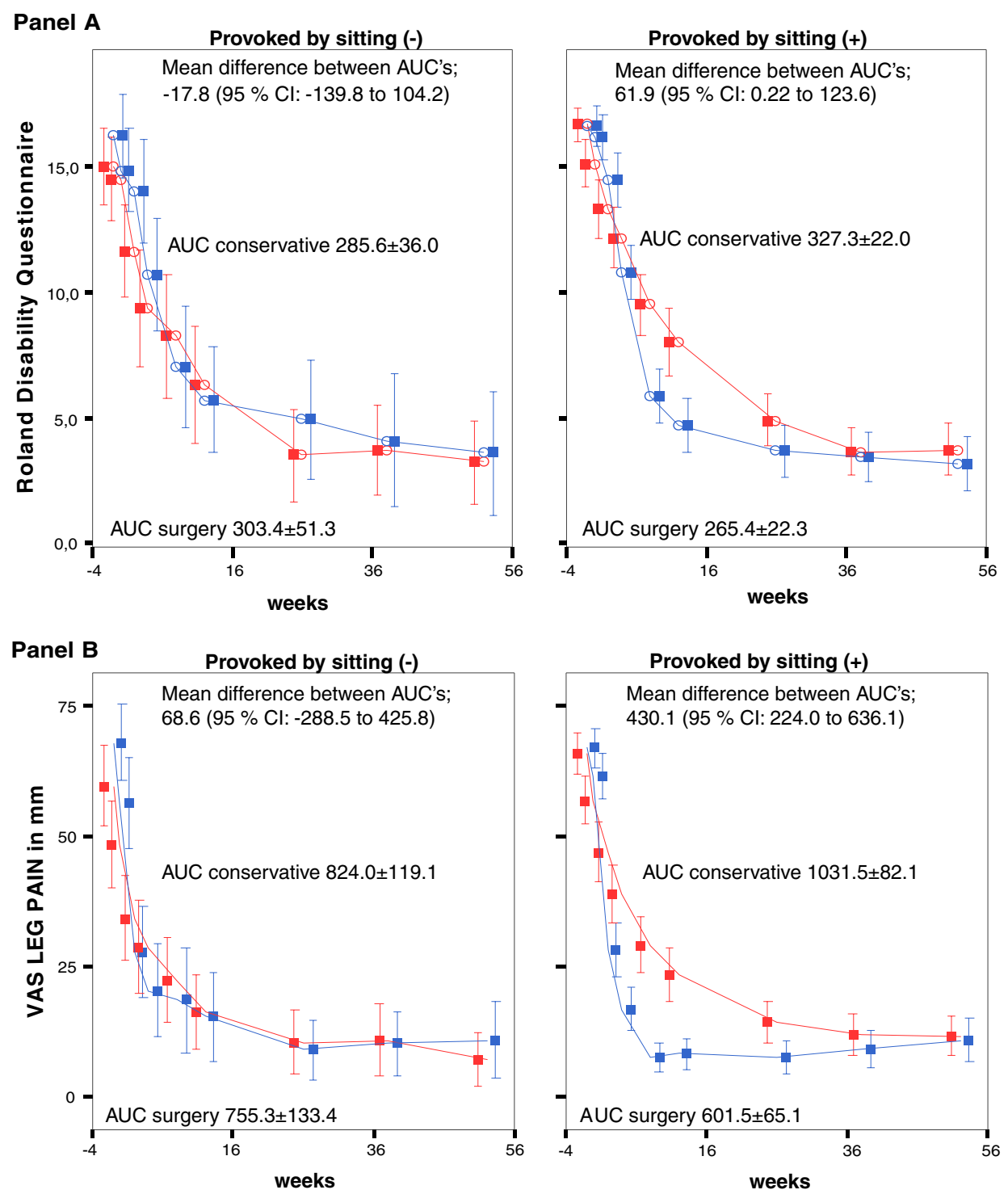

Panel C Provoked by sitting (-)
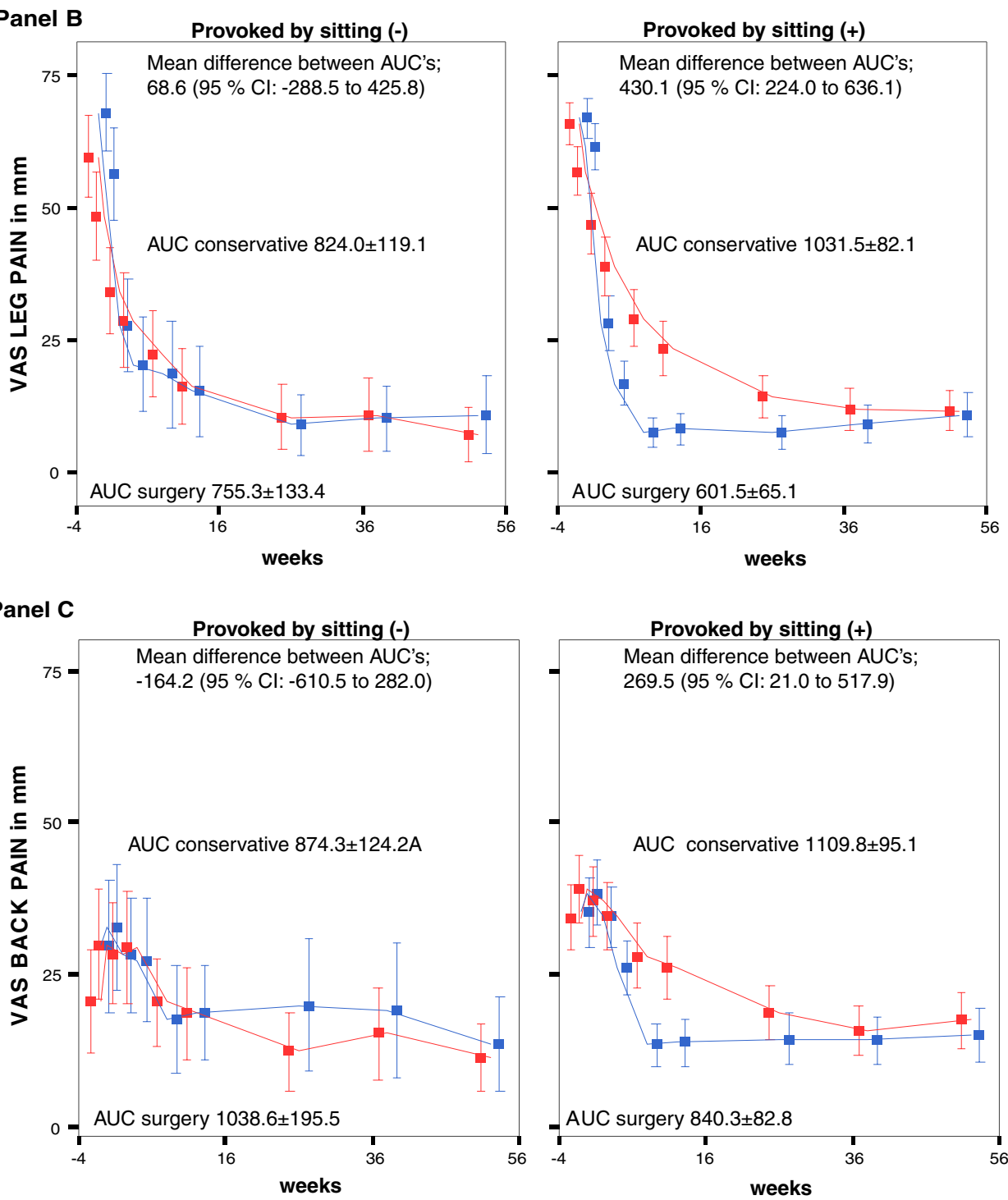

Provoked by sitting (+)

Mean difference between AUC's; 269.5 (95\% Cl: 21.0 to 517.9 )

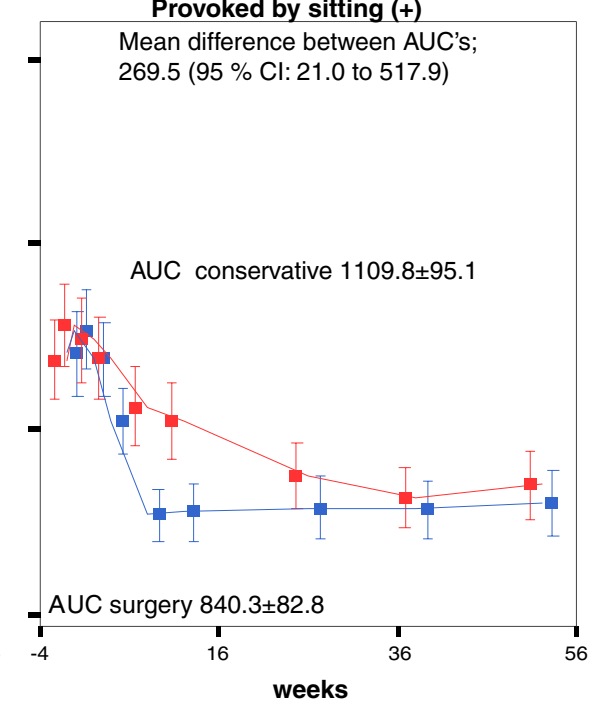


In contrast with our expectations sequestrated disc herniations also failed to follow a significantly different course when allotted to early surgery compared to prolonged conservative care. Earlier radiological studies showed strong associations between the type of disc herniation and the natural course or surgical outcome of sciatica [8, 23]. According to some authors sequestrated disc fragments were likely to resolve in the spinal epidural space, making surgery a pointless intervention [3, 4, 8]. Similar conclusions were drawn in the past in favour of MRI gadolinium rim enhancement of the disc herniation, representing neovascularization corresponding to macrophage resorption of the disc fragment $[2,10]$. The current study did not show any relationship between this variable and timing of surgery. Other important effectiveness studies suggested a relationship between spinal level of disc herniation and the surgical timing strategy. This was not confirmed by this analysis, which contains more solid data on duration of sciatica complaints and timing of surgery [25] and sample size [12].

While the scientific value of "sciatica provoked by sitting" as a prognostic variable might be debated, a similar result for this anamnestic variable was found in the randomized bed rest trial to predict the risk for patients undergoing surgery [22]. Although only a marginally significant interaction effect was found by the Cox proportional hazard analysis, these results appeared consistent when repeated measurement analysis of primary outcomes was performed. Furthermore it is a simple question to ask and physiologically completely understandable that a patient, persistently unable to sit, will gain important relief of pain, quality of life and function with early surgery. On the other hand if patients do not suffer sciatica provoked by sitting, their chances of a beneficial result of early surgery, if any, are reduced. Most of the latter patients might be better off with prolonged conservative care. Since this subgroup, however, was relatively small, one must interpret these results carefully; further investigation in future studies on this subject is needed.

The lack of a prognostic value of physical signs and symptoms for the outcome of sciatica has been reported before, but these studies focussed on the long-term results and not on the short-term rate of recovery [7, 9, 21]. It still is important to define neurological deficits [20] when examining a patient but their predictive value, to alter a decision to operate or to advise patients to stay conservative for a prolonged time, is minimal or absent. Nowadays, spine-oriented clinics request MRI quite early in the course of sciatica to comfort their patients and discuss treatment and prognosis. This study shows evidence of absent predictive and no prognostic value for this diagnostic strategy. MRI is necessary for surgery but is an expensive decision tool and less informative than a simple question asked during the triage of patients before deciding whether to refer for surgery or not. Well informed patients with high leg pain intensity and disability scores may be offered early surgery [16] especially when these baseline scores are combined with the inability to sit.

\section{Conclusion}

Except for absent "sciatica provoked by sitting" early surgery compared to prolonged conservative care yielded significantly faster rates of recovery for all investigated variables, irrespective of their value. Neurological signs, patient preferences and MRI findings fail to affect the outcome of early surgery versus prolonged conservative care. A simple question might help patients and spinal surgeons to decide for the optimal "timing of surgery" strategy.

Acknowledgments This study was funded by grants from the Netherlands Organisation for Health Research and DevelopmentZonMW and the Hoelen Foundation, The Hague to Wilco C. Peul [ISRCT 26872154]. They did have no role in the preparation of the protocol, design, analysis, synthesis and preparation of manuscript. We are indebted to Mrs Gail Bieger for reviewing the manuscript and the patients for participation in this study.

Conflict of interest statement We declare that we have no conflict of interest.

Open Access This article is distributed under the terms of the Creative Commons Attribution Noncommercial License which permits any noncommercial use, distribution, and reproduction in any medium, provided the original author(s) and source are credited.

\section{Appendix}

The participants of the Leiden-The Hague Spine Intervention Prognostic Study Group were as follows: Protocol Committee-W.C. Peul, B.W. Koes and R.T.W.M. Thomeer; Steering Committee-B.W. Koes, R.T.W.M. Thomeer, J.A.H. Eekhof, J.Th.J. Tans, W.B. van den Hout, W.C. Peul (principal investigator), R. Brand and H.C. van Houwelingen; Statistical analysis-R. Brand, W.C. Peul and H.C. van Houwelingen; Manuscript preparationW.C. Peul, B.W. Koes and R.T.W.M. Thomeer; Study Group, Recruitment and Data Collection and Management-M. Nuyten, P. Bergman, G. Holtkamp, S. Dukker, A. Mast, L. Smakman, C. Waanders, L. Polak and A. Nieborg; Participating hospitals and coordinating physicians-Medical Center Haaglanden, The Hague-J.Th.J. Tans and R. Walchenbach; Diaconessen Hospital, LeidenJ. van Rossum, P. Schutte and R.T.W.M. Thomeer; Groene Hart Hospital, Gouda-G.A.M. Verheul, J.E. Dalman and 
J.A.L. Wurzer; Reinier de Graaf Hospital, Delft/Voorburg-J.W.A. Sven and A. Kloet; Spaarne Hospital, Heemstede/Haarlem-I.S.J. Merkies and H. van Dulken; Bronovo Hospital, The Hague-P.C.L.A. Lambrechts and J.A.L. Wurzer; Haga Hospital, The Hague-R.W.M. Keunen and C.F.E. Hoffmann; Rijnland Hospital, Leiderdorp/Alphen ad Rijn-J. Haan and H. van Dulken; Lange Land Hospital, Zoetermeer-R. Groen and R.R.F. Kuiters; Leiden University Medical Center, Leiden-R.A.C. Roos and J.H.C. Voormolen; General Practitioners, Leiden/ Oegstgeest/Leiderdorp/Zoetermeer/Gouda/Delft/Heemstede/ Voorburg/Wassenaar/The Hague_-J.A.H. Eekhof.

\section{References}

1. Andersson GB, Brown MD, Dvorak J, Herzog RJ, Kambin P, Malter A, McCulloch JA, Saal JA, Spratt KF, Weinstein JN (1996) Consensus summary of the diagnosis and treatment of lumbar disc herniation. Spine 21:75S-78S

2. Autio RA, Karppinen J, Kurunlahti M, Haapea M, Vanharanta H, Tervonen O (2004) Effect of periradicular methylprednisolone on spontaneous resorption of intervertebral disc herniations. Spine 29(15):1601-1607

3. Carragee EJ, Kim DH (1997) A prospective analysis of magnetic resonance imaging findings in patients with sciatica and lumbar disc herniation. Correlation of outcomes with disc fragment and canal morphology. Spine 22:1650-1660. doi:10.1097/00007632199707150-00025

4. Carragee EJ, Han MY, Suen PW, Kim D (2003) Clinical outcomes after lumbar discectomy for sciatica: the effects of fragment type and anular competence. J Bone Joint Surg Am 85(1):102-108

5. Cherkin DC, Deyo RA, Loeser JD, Bush T, Waddell G (1994) An international comparison of back surgery rates. Spine 19:12011206. doi:10.1097/00007632-199406000-00001

6. Collins SL, Moore RA, McQuay HJ (1997) The visual analogue pain intensity scale: what is moderate pain in millimetres? Pain 72:95-97. doi:10.1016/S0304-3959(97)00005-5

7. Dvorak J, Valach L, Fuhrimann P, Heim E (1998) The outcome of surgery for lumbar disc herniation. II. A 4-17 years' follow-up with emphasis on psychosocial aspects. Spine 13(12):1423-1427

8. Ito T, Takano Y, Yuasa N (2001) Types of lumbar herniated disc and clinical course. Spine 26(6):648-651

9. Junge A, Dvorak J, Ahrens S (1995) Predictors of bad and good outcomes of lumbar disc surgery. A prospective clinical study with recommendations for screening to avoid bad outcomes. Spine 20:460-468. doi:10.1097/00007632-199502001-00009

10. Komori H, Okawa A, Haro H, Muneta T, Yamamoto H, Shinomiya K (1998) Contrast-enhanced magnetic resonance imaging in conservative management of lumbar disc herniation. Spine 23(1):67-73
11. Lutz GK, Butzlaff ME, Atlas SJ, Keller RB, Singer DE, Deyo RA (1999) The relation between expectations and outcomes in surgery for sciatica. J Gen Intern Med 14:740-744. doi:10.1046/j. 1525-1497.1999.10417.x

12. Osterman H, Seitsalo S, Karppinen J, Malmivaara A (2006) Effectiveness of microdiscectomy for lumbar disc herniation: a randomized controlled trial with 2 years of follow-up. Spine 31:2409-2414. doi:10.1097/01.brs.0000239178.08796.52

13. Patrick DL, Deyo RA, Atlas SJ, Singer DE, Chapin A, Keller RB (1995) Assessing health-related quality of life in patients with sciatica. Spine 20:1899-1908. doi:10.1097/00007632-199509000-00011

14. Peul WC, van Houwelingen HC, van der Hout WB, Brand R, Eekhof JA, Tans JT, Thomeer RT, Koes BW (2005) Prolonged conservative treatment or 'early' surgery in sciatica caused by a lumbar disc herniation: rationale and design of a randomized trial [ISRCT 26872154]. BMC Musculoskelet Disord 6:8. doi: 10.1186/1471-2474-6-8

15. Peul WC, van Houwelingen HC, van den Hout WB, Brand R, Eekhof JA, Tans JT, Thomeer RT, Koes BW (2007) Surgery versus prolonged conservative treatment for sciatica. N Engl $\mathrm{J}$ Med 356:2245-2256. doi:10.1056/NEJMoa064039

16. Peul WC, Brand R, Thomeer RT, Koes BW (2008) Improving prediction of "inevitable" surgery during non-surgical treatment of sciatica. Pain 138:571-576. doi:10.1016/j.pain.2008.02.011

17. Peul WC, Van Den Hout WB, Brand R, Thomeer RT, Koes BW (2008) Prolonged conservative care versus early surgery in patients with sciatica caused by lumbar disc herniation: two year results of a randomised controlled trial. BMJ 336:1355-1358. doi:10.1136/bmj.a143

18. Vader J-P, Porchet F, Larequi-Lauber T, Dubois RW, Burnand B (2000) Appropriateness of surgery for sciatica: reliability of guidelines from expert panels. Spine 25(14):1831-1836

19. van Tulder MW, Koes BW, Bouter LM (1995) A cost-of-illness study of back pain in The Netherlands. Pain 62:233-240. doi: 10.1016/0304-3959(94)00272-G

20. Vroomen PCAJ, de Krom MCTF, Knottnerus JA (1999) Diagnostic value of history and physical examination in patients suspected of sciatica due to disc herniation: a systematic review. J Neurol 246(10):899-906

21. Vroomen PCAJ, de Krom MCTF, Knottnerus JA, Fairbank J (2000) Consistency of history taking and physical examination in patients with suspected lumbar nerve root involvement. Spine 25(1):91-97

22. Vroomen PCAJ, de Krom MCTF, Knottnerus JA (2002) Predicting the outcome of sciatica at short-term follow-up. Br J Gen Pract 52(475):119-123

23. Vroomen PCAJ, Wilmink JT, de Krom MCTF (2002) Prognostic value of MRI findings in sciatica. Neuroradiology 44(1):59-63

24. Weber H (1983) Lumbar disc herniation. A controlled, prospective study with ten years of observation. Spine 8(2):131-140

25. Weinstein JN, Tosteson TD, Lurie JD, Tosteson AN, Hanscom B, Skinner JS, Abdu WA, Hilibrand AS, Boden SD, Deyo RA (2006) Surgical vs nonoperative treatment for lumbar disk herniation: the Spine Patient Outcomes Research Trial (SPORT): a randomized trial. JAMA 296:2441-2450. doi:10.1001/jama.296.20.2441 